\title{
Influence of higher sodium substitutions on magnetic entropy change and transition temperature in lanthanum manganites
}

\author{
N SETHULAKSHMI $^{1}$, I A AL OMARI ${ }^{2}$ and M R ANANTHARAMAN ${ }^{1, *}$ \\ ${ }^{1}$ Department of Physics, Cochin University of Science and Technology, Cochin 682022, Kerala, India \\ ${ }^{2}$ Department of Physics, Sultan Qaboos University, PC 123 Muscat, Sultanate of Oman
}

MS received 6 May 2015; accepted 23 July 2015

\begin{abstract}
Present investigation focuses on the variation of magnetic entropy change for higher sodium substitution above $50 \%$ in lanthanum manganites. Magnetic measurements indicated that all samples exhibit a ferromagnetic ordering near room temperature. Magnetic isotherms for different temperatures above and below $300 \mathrm{~K}$ were recorded and the magnetic entropy change for compositions belonging to $\mathrm{La}_{1-x} \mathrm{Na}_{x} \mathrm{MnO}_{3}$ for $x=0.6$ to 0.9 in steps of 0.1 was estimated for different applied magnetic fields. It has been found that the compositions having a Na substitution of 0.6 are having the largest entropy change, and the entropy change increases with applied magnetic field for all compositions. The change in entropy also decreases with increase in substitution of sodium. A notable change in transition temperature with Na concentration is also found in this sample series.
\end{abstract}

Keywords. Magnetic materials; manganites; magnetic entropy change.

\section{Introduction}

Manganites are perceived as interesting materials with strong correlation between charge and spin degrees of freedom. These classes of materials belong to the family of perovskites $\left(\mathrm{ABO}_{3}\right)$, possessing $\mathrm{BO}_{6}$ octahedra with $\mathrm{B}$ and $\mathrm{A}$ sites occupied by divalent, trivalent or tetravalent ions. Manganites, especially rare-earth manganites $\left(\mathrm{REMnO}_{3}\right)$, attracted immense attention after the discovery of colossal magnetoresistance. They are characterized by having one type spin electrons dominating over the other and only energies of these particular spin electrons reach the Fermi level. These materials thus belong to a specific class called half metallic compounds, as the material acts as an insulator for one type of spins while for other type, it is a conductor. Spindependent transport is a unique feature of these class of materials, which has been exploited largely for developing spintronic devices, a concept of utilizing the spin of electrons in addition to charge. ${ }^{1,2}$ Manganites also posses large magnetic moments and exhibit a variety of magnetic phases, such as ferromagnetism (FM), antiferromagnetism (AFM), charge ordering, spin glass or canted AFM. Magnetic property is largely dependent on exchange interactions between mixed valence states of $\mathrm{Mn}$ and is also closely linked to the spindependent electron transfer. From a fundamental perspective, they serve as ideal templates for studying correlation between structure, magnetic and charge transport. ${ }^{3,4}$

\footnotetext{
*Author for correspondence (mraiyer@yahoo.com)
}

Properties of manganites are dependent on size and valence states of $\mathrm{A}$ or $\mathrm{B}$ site ions, their concentration, nature of substituting elements for A and B ions, valence states of $\mathrm{Mn}$ and the oxygen stoichiometry. Variation in any of these parameters can result in structural changes, resulting in distortion in the form of deformation of unit cell from the ideal cubic phase. Structural distortion in manganites is often associated with Jahn teller distortion, which is a deciding factor for inducing further changes in magnetoresistance and formation of magnetic phases. There exist reports in literature dealing with hole- or electron-doped manganite systems with a wide variety of structural, magnetic and electrical properties. They are also known to influence electron transport. ${ }^{5-8}$

Magnetocaloric effect (MCE) is termed as the reversible change in temperature exhibited by materials with and without external magnetic field and such materials are called magnetocaloric materials. Manganites with large magnetic moments close to room temperature can serve as excellent candidates for magnetic refrigeration applications, which add to their multifunctionality. ${ }^{9,10}$ This phenomenon can be traced to the Maxwell's thermodynamic relations and adiabatic demagnetization. Magnetic refrigeration applications are characterized by magnetic entropy $\left(\Delta S_{\mathrm{M}}\right)$ and adiabatic temperature change $(\Delta T)$. MCE is an intelligent substitute for the conventional cooling technology, as it does not make use of any refrigerant gases and is an ecofriendly refrigeration mechanism. Large magnetic entropy changes have been observed so far for Gd-based compounds, such as $\mathrm{Gd}_{5}(\mathrm{SiGe})_{4}$ and $\mathrm{MnFe}$ alloys close to the mark of $40 \mathrm{~J} \mathrm{~kg}^{-1} \mathrm{~K}^{-1}$. ${ }^{11}$ Many other materials such as Laves phases 
$(\mathrm{La}(\mathrm{FeSi}))$, MnAs, heusler alloys and rare-earth manganites are also being increasingly investigated for magnetic refrigeration. ${ }^{12,13}$ Manganites have an advantage of tunable magnetic properties with respect to substituting elements and the potential of developing a near room temperature magnetic refrigerant. ${ }^{14}$

A survey of literature also reveals that though compositions having lower substitutions of sodium (Na) in $\mathrm{LaMnO}_{3}$ (LMO) have been investigated and thoroughly studied, there exists no report on higher sodium substitution above 50\%. For higher substitution levels of sodium, maintaining its exact stoichiometry in the compound is challenging as sodium is volatile. Thus sodium substitution in $\mathrm{LaMnO}_{3}$ presents new challenges, especially from the sample preparation point of view because of the loss of $\mathrm{Na}$ during sintering or synthesis. As an initial study, the composition with $50 \% \mathrm{Na}$ substitution (LNMO5) was prepared. Investigation carried out on LNMO5 for evaluating the $\Delta S_{\mathrm{M}}$ was encouraging and it has been found that $50 \% \mathrm{Na}$-substituted LMO is a potential magnetic refrigerant. ${ }^{15}$ It could then be presumed that compositions with $\mathrm{Na}$ above $50 \%$, i.e., $\mathrm{La}_{1-x} \mathrm{Na}_{x} \mathrm{MnO}_{3}$ $(x=0.6$ to 0.9$)$, are also of interest. Present work is a systematic study on magnetic characteristics and entropy changes associated with LMO for $\mathrm{Na}$ compositions greater than $50 \%$. Monovalent substitution at La sites can lead to the formation of twice the number of holes than divalent substitution, which can play a decisive role in the conversion of $\mathrm{Mn}^{3+}$ ions to $\mathrm{Mn}^{4+}$ ions, thereby in the magnetic property. ${ }^{16}$ Monovalent ions like $\mathrm{K}, \mathrm{Li}$ have been substituted for A site rare-earth atoms and recently $\mathrm{Na}$ in $\mathrm{PrSrMnO}_{3}$ has been reported to exhibit $\Delta S_{\mathrm{M}}$ of $4.4 \mathrm{~J} \mathrm{~kg}^{-1} \mathrm{~K}^{-1}$ at $290 \mathrm{~K}$ at an applied external magnetic field of $7 \mathrm{~T}^{17}$ Sodium substitution in $\mathrm{LaBaMnO}_{3}$ has led to a $\Delta S_{\mathrm{M}}$ of $4 \mathrm{~J} \mathrm{~kg}^{-1} \mathrm{~K}^{-1}$ at 280 $\mathrm{K}$ for $7 \mathrm{~T}$ external magnetic field. ${ }^{18}$ There are also reports to suggest that a magnetic entropy change of $3 \mathrm{~J} \mathrm{~kg}^{-1} \mathrm{~K}^{-1}$ was observed in lower sodium-substituted manganite, that is, $\mathrm{La}_{0.65} \mathrm{Ca}_{0.3} \mathrm{Na}_{0.05} \mathrm{MnO}_{3}$ below $300 \mathrm{~K} .{ }^{19}$ Magnetocaloric studies carried out in $\mathrm{La}_{0.799} \mathrm{Na}_{0.199} \mathrm{Mn}_{1.000} \mathrm{O}_{2.97}$ by Zhong et al revealed a $\Delta S_{\mathrm{M}}$ of $1.96 \mathrm{~J} \mathrm{~kg}^{-1} \mathrm{~K}^{-1}$ at $334 \mathrm{~K}$. A magnetic entropy change within a range of $1-3 \mathrm{~J} \mathrm{~kg}^{-1} \mathrm{~K}^{-1}$ was observed for lower levels of monovalent ion substitutions ( $\mathrm{Na}$ or $\mathrm{K}$ ) in lanthanum manganites. ${ }^{20,21}$

\section{Experimental}

Sodium ion $\left(\mathrm{Na}^{+}\right)$is substituted for $\mathrm{La}^{3+}$ ion in LMO as ionic radii of $\mathrm{Na}^{+}$is comparable with $\mathrm{La}^{3+}$ thereby limiting structural distortion. Samples with $\mathrm{Na}$ concentration of 0.6 to 0.9 in steps of 0.1 (samples coded as LNMO6 to LNMO9 correspondingly, shortly as LNMO series) were synthesized using citrate gel method. Required amounts of lanthanum oxide, manganese nitrate and sodium carbonate were dissolved in de-ionized water. Adequate amount of citric acid and nitric acid was added to the solution and was heated to a temperature of $80^{\circ} \mathrm{C}$ with continuous stirring. The resultant dark powder was then fired at a temperature of $1100^{\circ} \mathrm{C}$ for several hours. Structural studies were carried out using X-ray diffraction and magnetization studies were carried out using a Vibrating Sample Magnetometer (VSM) and a SQUID Magnetometer. Magnetization data were utilized to evaluate the magnetic entropy change.

\section{Results and Discussion}

All the four samples of substituted lanthanum manganite, namely, LNMO6, LNMO7, LNMO8 and LNMO9 exhibited distorted orthorhombic structure with a pbnm space group, which has been reported elsewhere. ${ }^{22}$ It has been reported that for $\mathrm{Na}$ substitutions ranging from 0.025 to 0.25 in LMO, samples exhibited rhombohedral structure with $R \overline{3} C$ space group. ${ }^{16}$ For $\mathrm{Na}$ substitutions in $\mathrm{La}$ and $\mathrm{Mn}$ sites in LMO system, rhombohedral structure was observed. A systematic decrease in rhombohedral distortion has been observed for sodium-substituted LMO system. ${ }^{23,24}$ Reports on sodium-substituted praseodymium manganites exhibiting orthorhomic structure with pbnm space group, showed an increase in orthorhombic distortion with decrease in $\mathrm{Na}$ ion concentration resulting in decrease of $\mathrm{Mn}-\mathrm{O}-\mathrm{Mn}$ bond angle and cell volume. Orthorhombic and rhombohedral phases have been observed in Na-substituted mixed manganites of praseodymium and lanthanum. ${ }^{24}$ Roy et $a l^{3}$ have reported a change in characteristic peak as the $\mathrm{Na}$ concentration is increased in manganites, indicating the onset of orthorhombic phase. Shimura et $a l^{4}$ have also proposed a distorted perovskite structure for monovalent ion doping. Magnetization measurements carried out using VSM (figure 1) indicated that all samples show ferromagnetic ordering with negligible coercivity. It is also found that saturation magnetization $\left(M_{\mathrm{S}}\right)$ decreases with increase in $\mathrm{Na}$ concentration. Magnetic ordering in manganites is mainly attributed to exchange mechanism between mixed valence states of $\mathrm{Mn} ; \mathrm{Mn}^{3+}$ and $\mathrm{Mn}^{4+}$. An X-ray photoelectron spectroscopy (XPS) profile of Mn2 $\mathrm{p}_{3 / 2}$ peak for LNMO sample series in figure 2 clearly indicates the presence of $\mathrm{Mn}^{3+}$ and $\mathrm{Mn}^{4+}$ ions and their variation with respect to Na concentration. It is found that as $\mathrm{Na}$ concentration increases, $\mathrm{Mn}^{4+}$ ions outnumber $\mathrm{Mn}^{3+}$ ions. Increase in $\mathrm{Mn}^{4+}$ ions result in a decreased double exchange interaction, which could lead to decrease in saturation magnetization. Reduced mobility of charge carriers owing to the presence of oxygen vacancies also has direct influence in magnetization decrease. ${ }^{25-27}$ Structual and magnetic studies of LNMO series do not form the objective of present article and have been discussed in another article of the same author. ${ }^{22}$ As part of a brief overview, two major results are indicated in figures 1 and 2. From figure 1 it is clear that samples are ferromagnetic at $300 \mathrm{~K} \cdot{ }^{15,22}$ Inset of figure 1 shows that all samples possess a nominal coercivity close to 10 Oe. Figure 2 indicates the Mn2 $\mathrm{p}_{3 / 2}$ XPS profile of LNMO series, which indicates the presence of $\mathrm{Mn}^{3+} / \mathrm{Mn}^{4+}$ ions and it is observed that as $\mathrm{Na}$ concentration increases, more $\mathrm{Mn}^{3+}$ ions are getting converted to $\mathrm{Mn}^{4+}$ ions. ${ }^{22}$ 


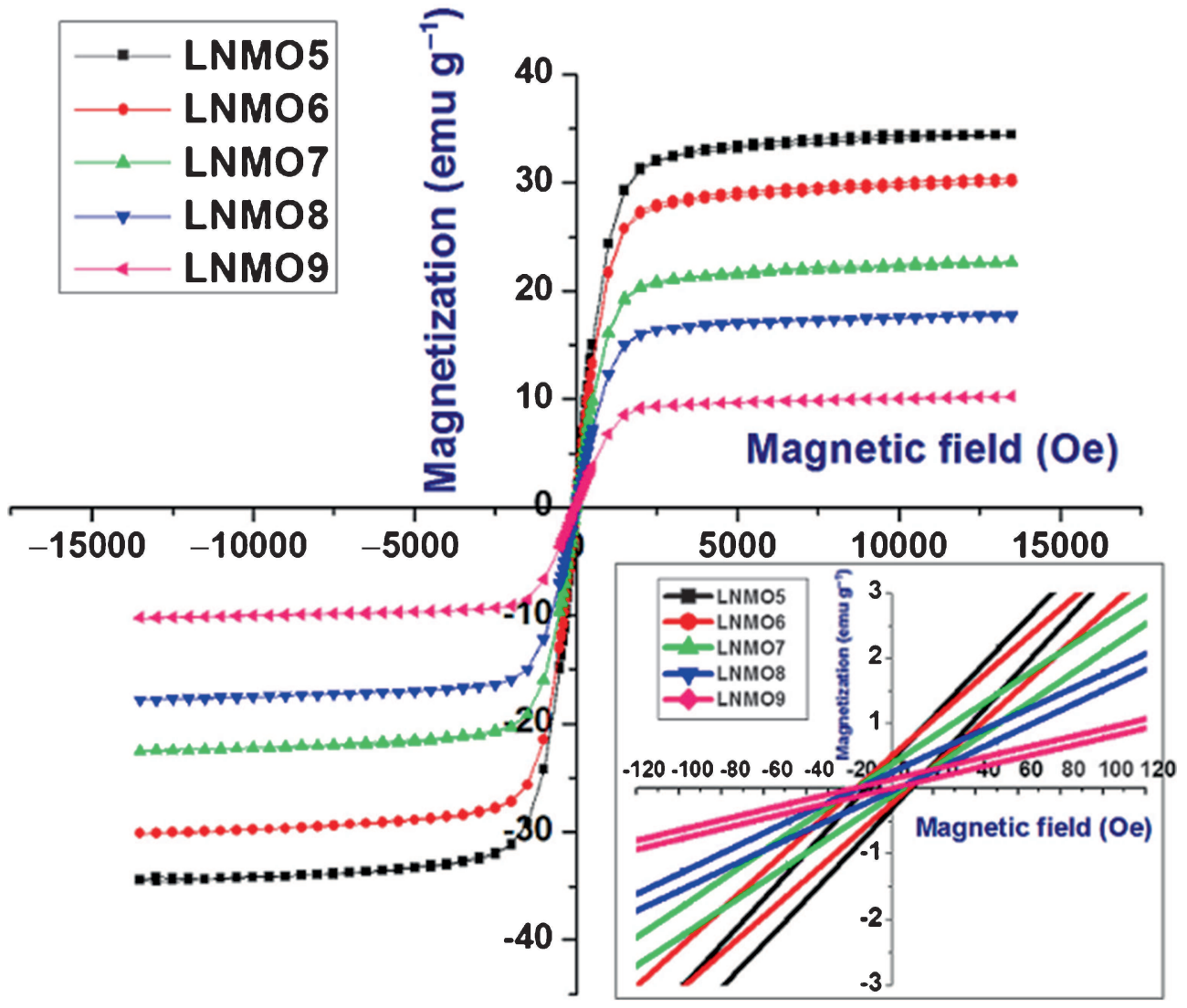

Figure 1. Ferromagnetic behaviour of LNMO samples. Inset is the magnified graph for the magnetic field range -120 to +120 Oe, which shows that coercivity is close to 10 Oe.

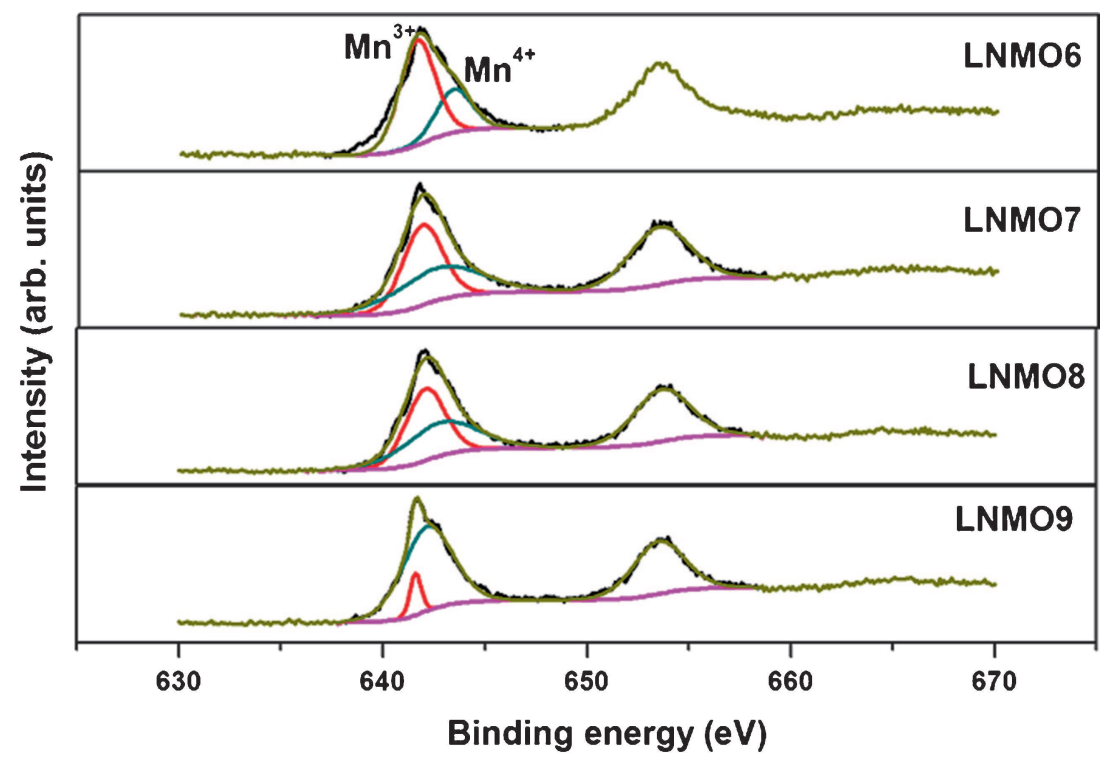

Figure 2. $\mathrm{Mn} 2 \mathrm{p}_{3 / 2}$ XPS profile of LNMO samples.

$M-H$ (magnetization-magnetic field) isotherms with magnetization expressed in $\mathrm{Am}^{2} \mathrm{~kg}^{-1}$ for magnetic field variation $\mu_{0} H$ up to $5 \mathrm{~T}$ in the temperature range $200-300 \mathrm{~K}$ in steps of $20 \mathrm{~K}$ for samples LNMO6, LNMO7, LNMO8 and LNMO9 are shown in figure 3.
Determination of magnetic entropy change from magnetic isotherms is a reliable technique, as it is difficult to determine the adiabatic temperature change directly in manganites. Area between $M-H$ isotherms is a measure of the entropy change and is governed by equation (1), which stems 

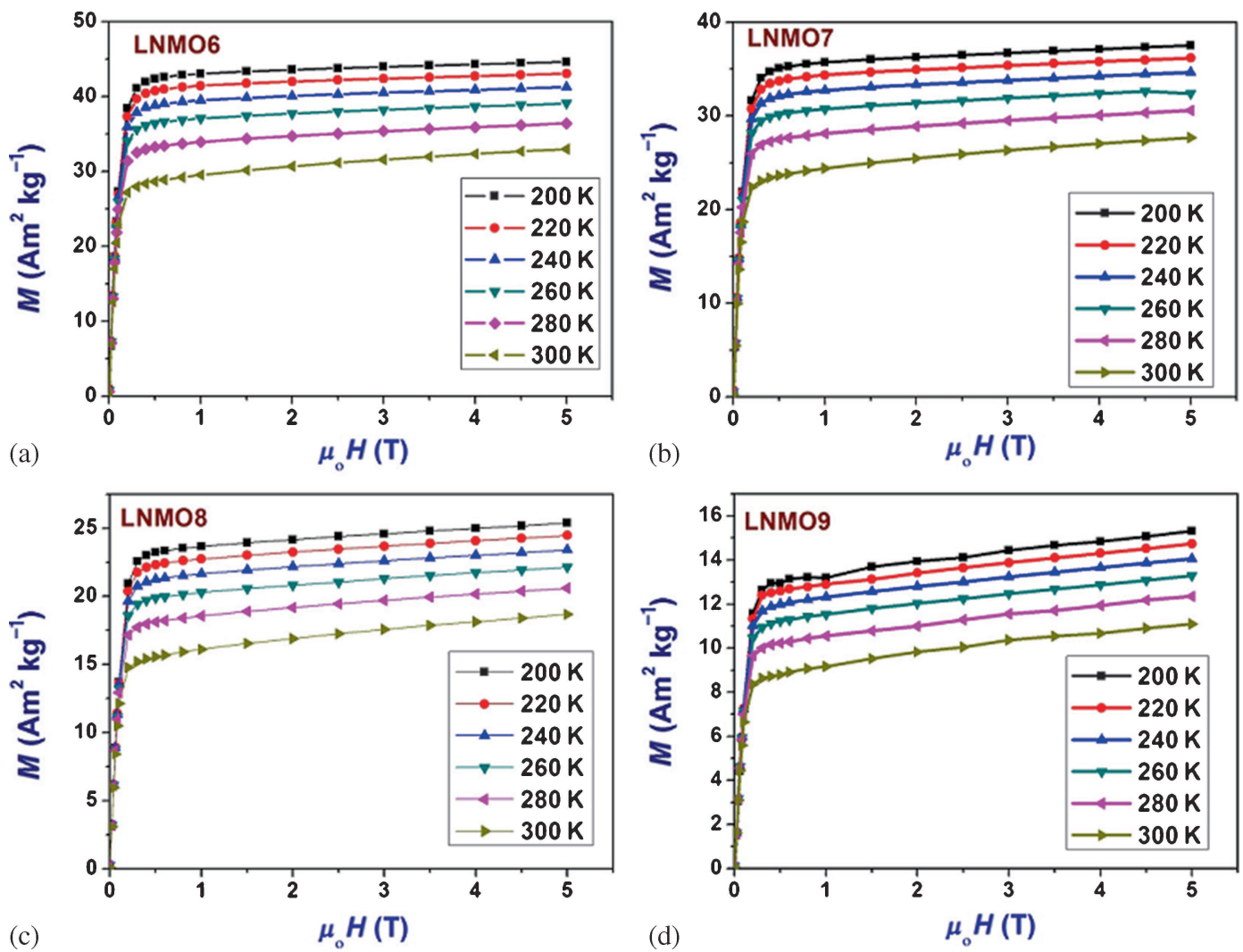

Figure 3. $\quad M-H$ isotherms for LNMO samples: (a-d) LNMO6 to LNMO9.

out from Maxwell's thermodynamic relations, ${ }^{28}$ where $M$ is the magnetization at a particular temperature $T$ and $H$ the magnetic field. An alternate form of equation (1) is given by equation (2), where $M_{i}$ and $M_{i+1}$ are the magnetization values corresponding to temperatures $T_{i}$ and $T_{i+1}$, respectively.

$$
\begin{aligned}
& \Delta S_{\mathrm{M}}(T, H)=\mu_{0} \int_{0}^{H} \frac{\partial M(T, H)}{\partial T} \mathrm{~d} H \\
& \Delta S_{\mathrm{M}}=\frac{\mu_{0}}{\Delta T}\left[\int_{0}^{H} M_{i+1}\left(T_{i+1}, H\right) \mathrm{d} H-\int_{0}^{H} M_{i}\left(T_{i}, H\right) \mathrm{d} H\right]
\end{aligned}
$$

Thus as per equation (2), entropy change, $\Delta S_{\mathrm{M}}$ can be evaluated by multiplying the reciprocal of temperature difference $(\Delta T)$ and area between two adjacent $M-H$ curves. ${ }^{28,29}$ Area between $200 \mathrm{~K}$ and $220 \mathrm{~K} M-H$ curves with $\Delta T$ of $20 \mathrm{~K}$ yields $\Delta S_{\mathrm{M}}$ value at $210 \mathrm{~K}$.

From the isotherms in the temperature range of 200 to $300 \mathrm{~K}$, it could be seen that all samples are ferromagnetic with a magnetic ordering temperature above $300 \mathrm{~K}$. Calculated magnetic entropy change $\left(\Delta S_{\mathrm{M}}\right)$ values as per equation (1) (absolute values, i.e., sign of entropy are not considered) for LNMO6 are shown in figure 4a. From figure $4 \mathrm{a}$ it is observed that $\Delta S_{\mathrm{M}}$ increases with increase in temperature reaching a maximum of $1 \mathrm{~J} \mathrm{~kg}^{-1} \mathrm{~K}^{-1}$ at $290 \mathrm{~K}$ and the entropy change increases with increase in external magnetic field. Entropy values for 0.5, 1, 2 and $5 \mathrm{~T}$ follow a decreasing trend for a particular temperature. For analysing entropy variation above $300 \mathrm{~K}$, magnetic isotherms in the high temperature range of 303 to $393 \mathrm{~K}$ were also analysed and the magnetic entropy variation was estimated. For this set of isotherms small field variations are considered, as main focus is on variation of entropy with temperatures above $300 \mathrm{~K}$.

Figure $4 \mathrm{~b}$ shows entropy variation in the temperature range 303-393 $\mathrm{K}$ for $0.5,1,1.15,1.25$ and $1.35 \mathrm{~T}$ magnetic field ranges. It indicates that $\Delta S_{\mathrm{M}}$ is found to be decreasing from $308 \mathrm{~K}$ and reaches a minimum at $388 \mathrm{~K}$. Thus the entropy variation is maximum in the temperature range of 250 to $320 \mathrm{~K}$. Entropy change at $308 \mathrm{~K}$ for $1.35 \mathrm{~T}$ magnetic field is found to be $0.082 \mathrm{~J} \mathrm{~kg}^{-1} \mathrm{~K}^{-1}$. Similarly, $\Delta S_{\mathrm{M}}$ values for LNMO7 is also determined and shown in figure 5a for 0.5, 1,2 and $5 \mathrm{~T}$ in the temperature range $200-300 \mathrm{~K}$, which also showed a decreasing trend with increase in temperature, a maximum $\Delta S_{\mathrm{M}}$ of $0.82 \mathrm{~J} \mathrm{~kg}^{-1} \mathrm{~K}^{-1}$ for $5 \mathrm{~T}$ magnetic field. For LNMO7, a maximum $\Delta S_{\mathrm{M}}$ of $0.028 \mathrm{~J} \mathrm{~kg}^{-1} \mathrm{~K}^{-1}$ is observed for $1.35 \mathrm{~T}$ as analysed from isotherms in the high temperature range 303-393 $\mathrm{K}$ (figure 5b). LNMO8 and LNMO9 recorded a maximum $\Delta S_{\mathrm{M}}$ of 0.55 and $0.32 \mathrm{~J} \mathrm{~kg}^{-1} \mathrm{~K}^{-1}$, respectively, for $5 \mathrm{~T}$ magnetic field (figures $6 \mathrm{a}$ and $7 \mathrm{a}$ ) in the temperature range $200-300 \mathrm{~K}$. In the high temperature range 303$393 \mathrm{~K}, \mathrm{LNMO} 8$ and LNMO9 possessed a $\Delta S_{\mathrm{M}}$ of 0.033 and 

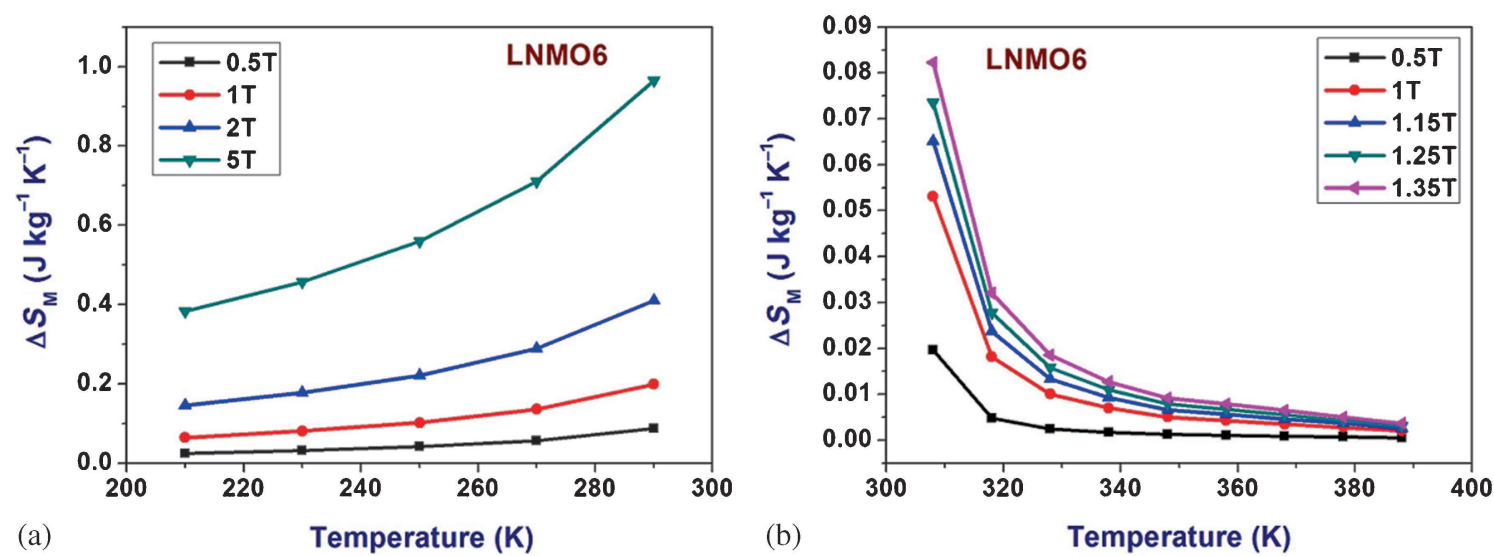

Figure 4. Magnetic entropy change $\left(\Delta S_{\mathrm{M}}\right)$ for LNMO6 sample for the temperature range (a) 200-300 K and (b) $303-393 \mathrm{~K}$.
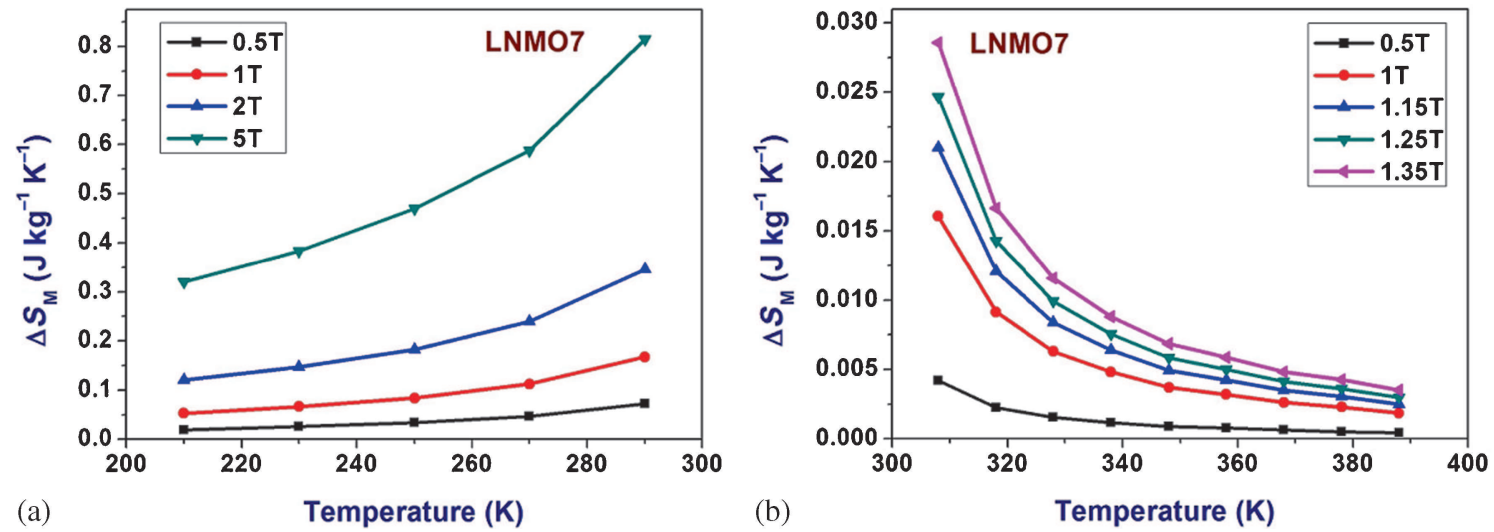

Figure 5. Magnetic entropy change $\left(\Delta S_{\mathrm{M}}\right)$ for LNMO7 sample for the temperature range (a) 200-300 K and (b) 303-393 K.
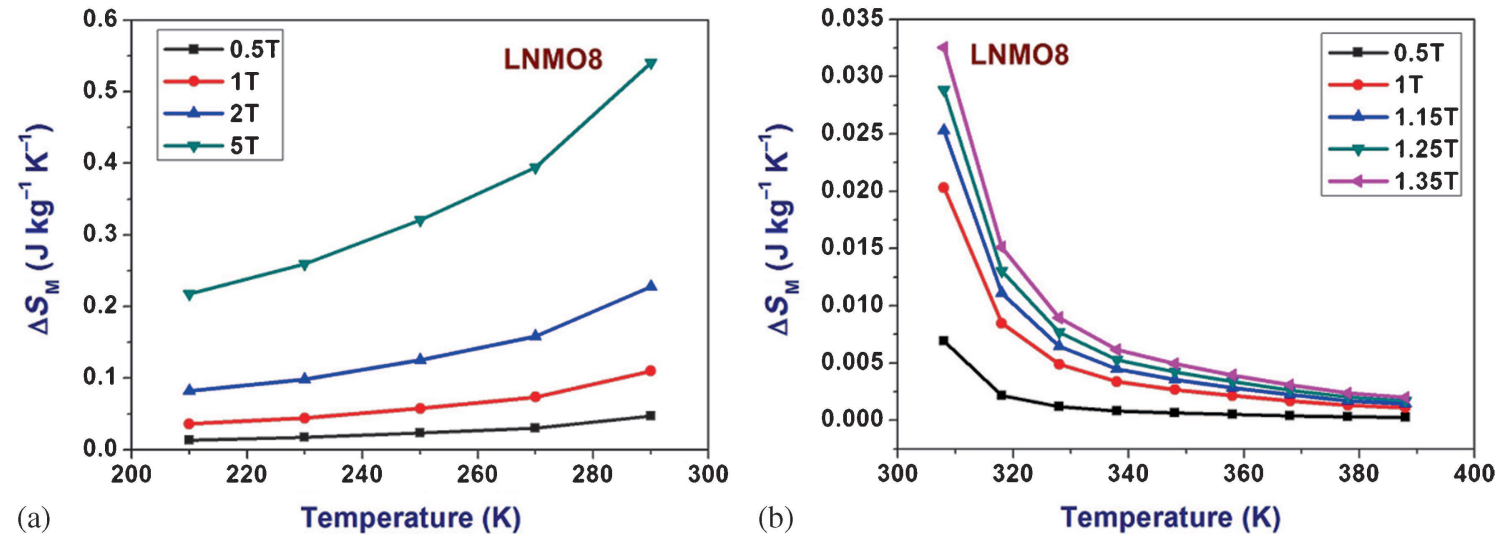

Figure 6. Magnetic entropy change $\left(\Delta S_{\mathrm{M}}\right)$ for LNMO8 sample for the temperature range (a) 200-300 K and (b) 303-393 K.

$0.015 \mathrm{~J} \mathrm{~kg}^{-1} \mathrm{~K}^{-1}$ for $1.35 \mathrm{~T}$ magnetic field, respectively (figures $6 \mathrm{~b}$ and $7 \mathrm{~b}$ ).

Entropy variation in the range 200-300 K and 303-393 K is depicted in figures 8 and 9, respectively. Maximum entropy variation is observed for LNMO6 and as Na content increases, value of entropy change decreases.

There are reports on magnetic entropy changes with respect to substituted lanthanum manganites. Zhong et al ${ }^{30}$ 

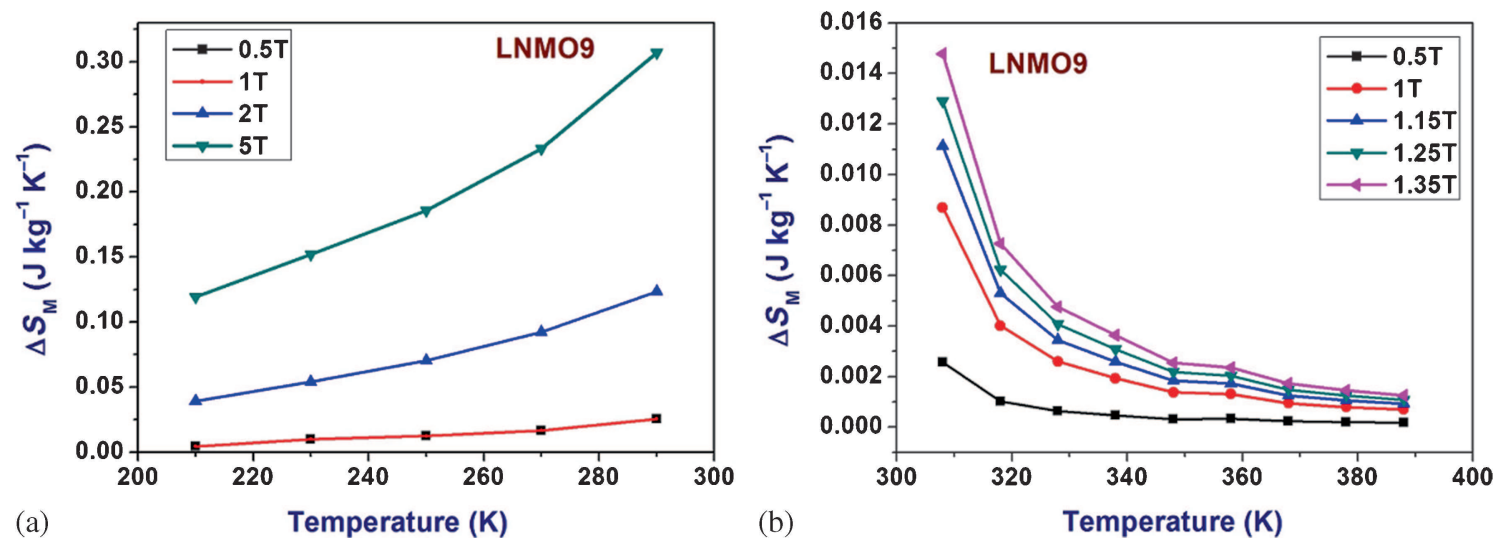

Figure 7. Magnetic entropy change $\left(\Delta S_{\mathrm{M}}\right)$ for LNMO9 sample for the temperature range (a) $200-300 \mathrm{~K}$ and (b) 303-393 K.

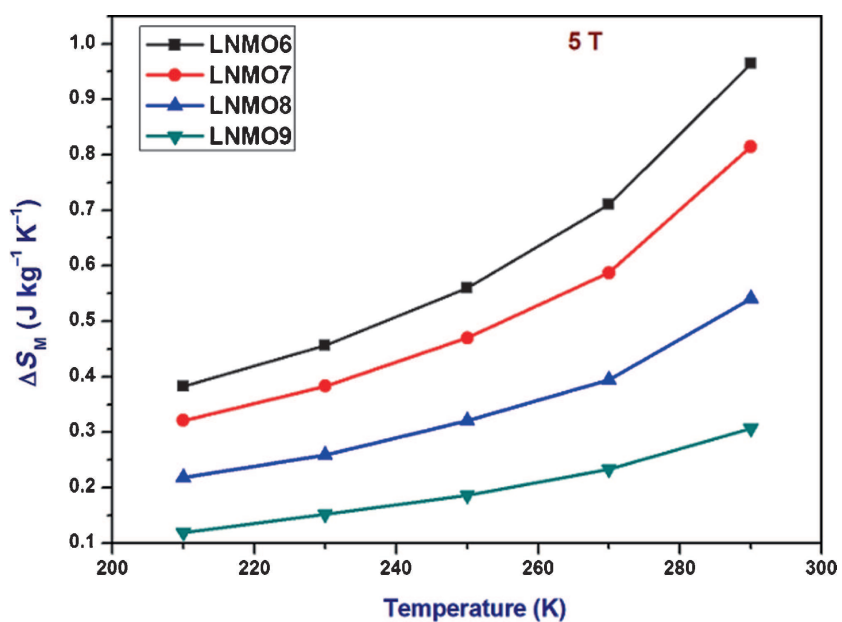

Figure 8. Magnetic entropy change $\left(\Delta S_{\mathrm{M}}\right)$ for LNMO6 to LNMO9 samples for the temperature range 200-300 K.

reported a $\Delta S_{\mathrm{M}}$ of 1.32 and $1.53 \mathrm{~J} \mathrm{~kg}^{-1} \mathrm{~K}^{-1}$ at 195 and $218 \mathrm{~K}$ under $1 \mathrm{~T}$ magnetic field for $\mathrm{La}_{0.925} \mathrm{Na}_{0.075} \mathrm{MnO}_{3}$ and $\mathrm{La}_{0.9} \mathrm{Na}_{0.1} \mathrm{MnO}_{3}$, respectively. But in both cases, magnetic ordering temperature was much below room temperature. However it must be noted that in the present LNMO series, entropy change obtained is maximum within the temperature range of 250 and $320 \mathrm{~K}$. In an earlier study conducted on $\mathrm{La}_{0.83} \mathrm{Li}_{0.17} \mathrm{Ti}_{0.4} \mathrm{Mn}_{0.6} \mathrm{O}_{3}$ a $\Delta S_{\mathrm{M}}$ of $0.9 \mathrm{~J} \mathrm{~kg}^{-1} \mathrm{~K}^{-1}$ was observed at $3 \mathrm{~T}$, while a $\Delta S_{\mathrm{M}}$ of $1.1 \mathrm{~J} \mathrm{~kg}^{-1} \mathrm{~K}^{-1}$ was recorded in $\mathrm{La}_{0.85} \mathrm{Li}_{0.15} \mathrm{Ti}_{0.3} \mathrm{Mn}_{0.7} \mathrm{O}_{3}$ at $3 \mathrm{~T}$ magnetic field at $80 \mathrm{~K}^{31}$ Thus major outcome of the present investigation is that entropy variation observed in all four samples belonging to the series $\mathrm{La}_{1-x} \mathrm{Na}_{x} \mathrm{MnO}_{3}$ is near room temperature with lower hysteresis loss or lower coercivity. The low values observed in these samples could be attributed to (a) distortion of lattice from orthorhombic to rhombohedral along with change in space group, and (b) presence of first-order magnetic transition from AFM to FM in addition to dominant second-order transition from FM to paramagnetism. It could be interesting to study the magnetic properties and

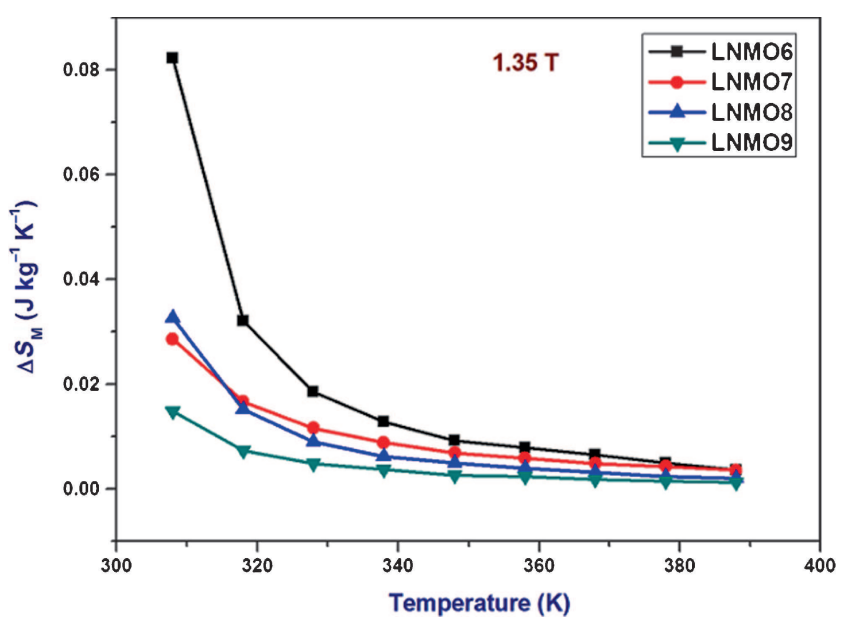

Figure 9. Magnetic entropy change $\left(\Delta S_{\mathrm{M}}\right)$ for LNMO6 to LNMO9 samples for the temperature range 303-393 K.

MCE behaviour in managnite systems, where monovalent as well as divalent ions are substituted for La sites.

Magnetic isotherms in the case of sample series of LNMO were also utilized to generate Arrott plots, which plots second-order terms in Landau free energy terms, i.e, $\mu_{0} H / M$ vs. $M^{2}$. Positive slope of Arrott plots are indicative of second-order magnetic transition. Arrott plots for LNMO series are shown in figures $10 \mathrm{a}, \mathrm{b}$ and $11 \mathrm{a}, \mathrm{b}$, which confirm that magnetic transition in them is of second order. In the Arrott plots, curve that can be extrapolated to origin corresponds to $T_{\mathrm{C}}$. For determining $T_{\mathrm{C}}$, a linear line from the origin that is $45^{\circ}$ inclined to both $x$ and $y$ axes are taken as reference line and the curve from isotherms corresponding to a particular temperature that coincides with the reference line gives the $T_{\mathrm{C}}$ value. In case if the reference line does not coincide but pass between two temperature curves, only the range of $T_{\mathrm{C}}$ could be specified. In our case for LNMO6 (figure 10a), the reference line passes between the two curves 333 and $323 \mathrm{~K}$ and hence $T_{\mathrm{C}}$ range is $333-323 \mathrm{~K}$. Also for LNMO7 sample (figure 10b), the reference line passes between the two curves 

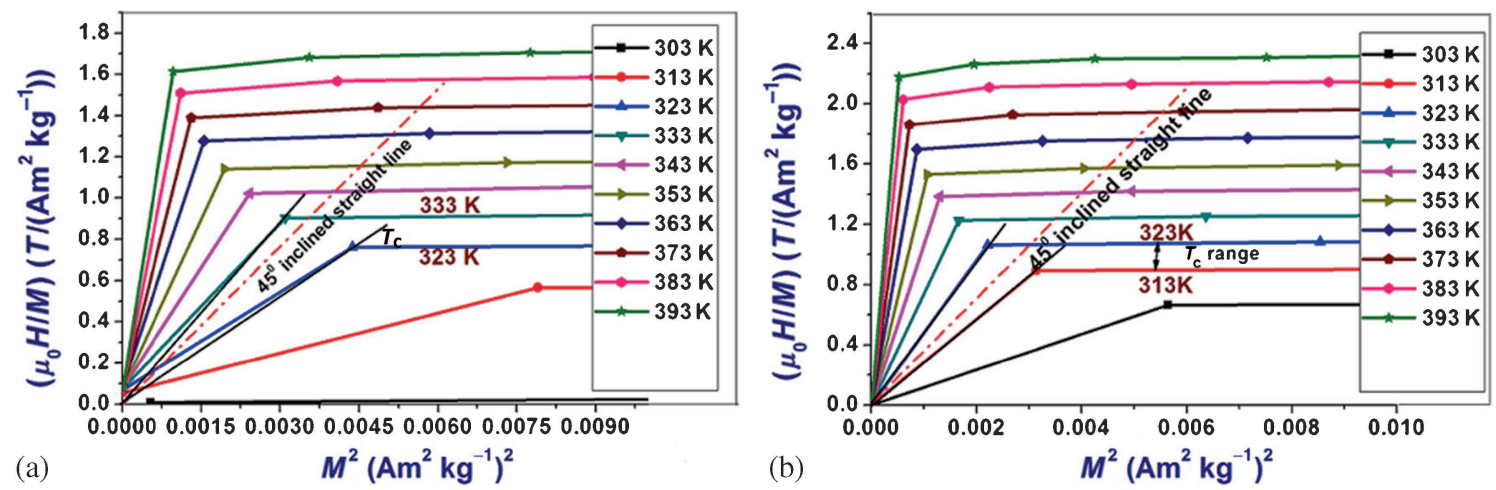

Figure 10. Determination of $T_{\mathrm{C}}$ range from Arrott plots: (a) for LNMO6 $T_{\mathrm{C}}$ range is between 333 and $323 \mathrm{~K}$ and (b) for LNMO7 $T_{\mathrm{C}}$ range is between 323 and $313 \mathrm{~K}$.
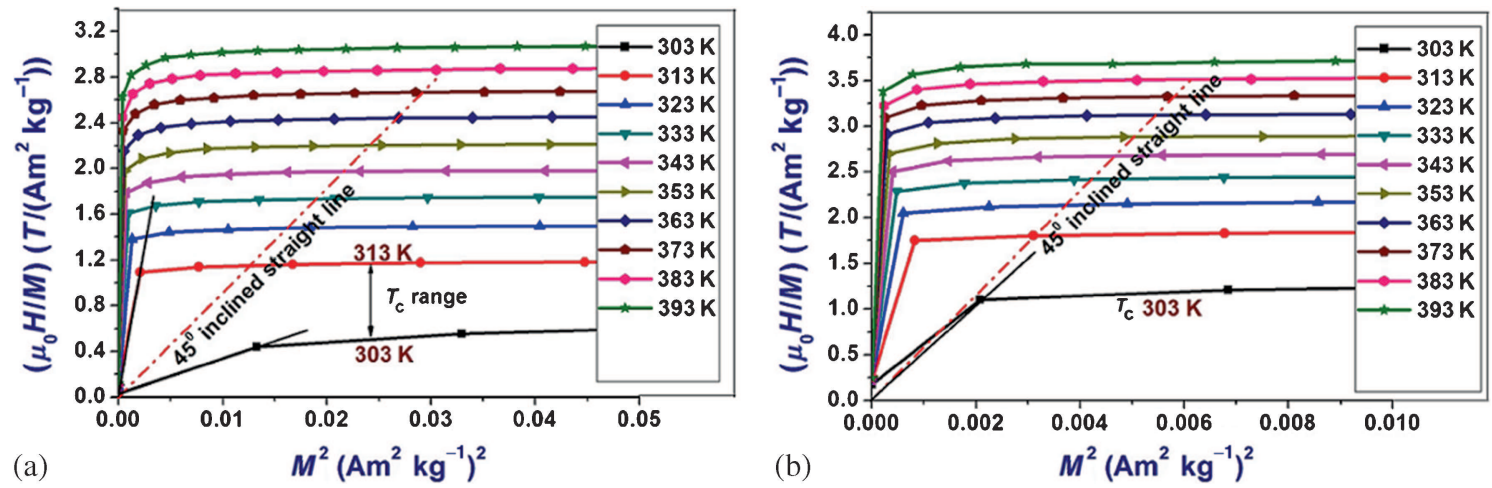

Figure 11. Determination of $T_{\mathrm{C}}$ range from Arrott plots: (a) for LNMO8 $T_{\mathrm{C}}$ range is between 313 and $303 \mathrm{~K}$ and (b) for LNMO9 $T_{\mathrm{C}}$ is at $303 \mathrm{~K}$.
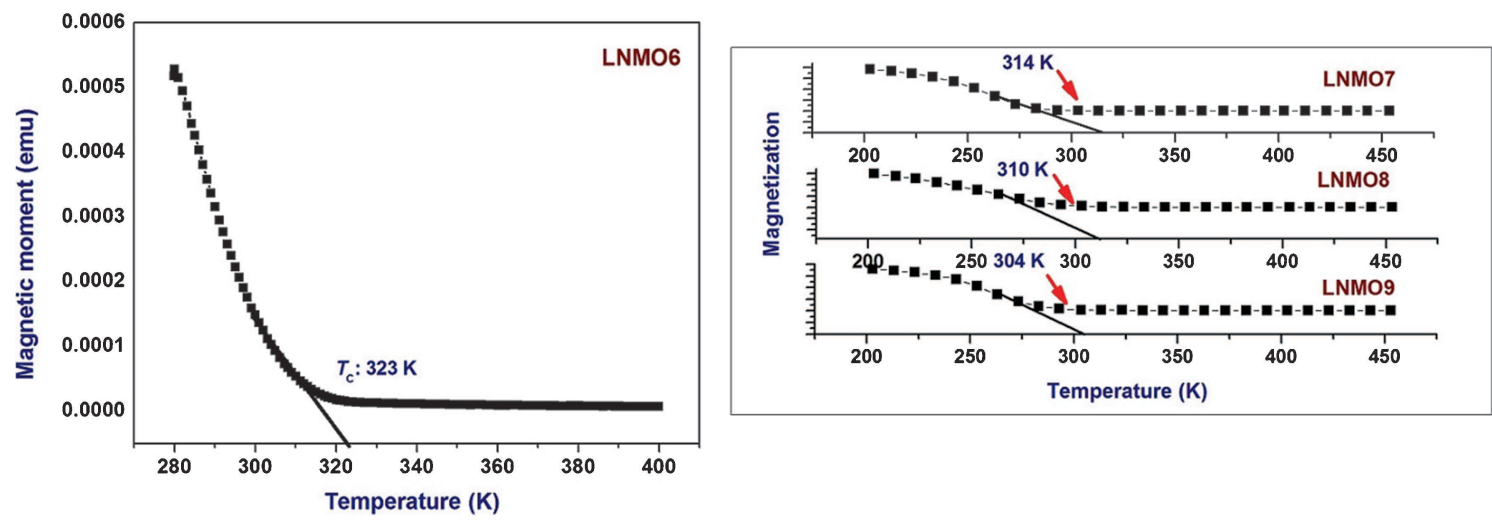

Figure 12. (a) $M$ vs. $T$ measurement for LNMO6, which indicates $T_{\mathrm{C}}$ at $323 \mathrm{~K}$. (b) $M v s$. $T$ measurement for LNMO7, LNMO8 and LNMO9 with $T_{\mathrm{C}}$ at 314,310 and $304 \mathrm{~K}$, respectively.

323 and $313 \mathrm{~K}$ and $T_{\mathrm{C}}$ range can be defined between 313 and $323 \mathrm{~K}$. In the case of LNMO8 (figure 11a) also, $T_{\mathrm{C}}$ range is between 313 and $303 \mathrm{~K}$ and in LNMO9 (figure $11 \mathrm{~b}$ ), $T_{\mathrm{C}}$ is found to be at around $303 \mathrm{~K}$. Arrott plots for LNMO7 and LNMO8 (figures $10 \mathrm{~b}$ and $11 \mathrm{a}$, respectively) are linear, while for LNMO9 (figure 11b) curves are slightly deviated from linear behaviour in the temperature range 200 to $300 \mathrm{~K}$ indicative of a magnetic inhomogeneity.
Estimation of exact value of $T_{\mathrm{C}}$ from Arrott plots is difficult as the curves overlap at high temperatures, but it can be established that there is considerable variation in $T_{\mathrm{C}}$ with $\mathrm{Na}$ doping and it decreases with increase in $\mathrm{Na}$ doping. Such anomalous behaviour in $T_{\mathrm{C}}$ from divalent ion substitutions has been observed earlier. It has been proposed that many factors like competition between different structures like orthorhombic or rhomohedral phases, Hund's 
coupling and oxygen vacancies could decide magnetic ordering mechanism and transition temperature. ${ }^{26,27}$ Magnetization $v s$. temperature measurements were also carried out on LNMO samples to determine transition temperature, $T_{\mathrm{C}}$, and it matches well with that obtained from Arrott plots. Figure 12a shows magnetization vs. temperature $(M v s . T)$ measurements on LNMO6, which indicates $T_{\mathrm{C}}$ at $323 \mathrm{~K}$, while figure $12 \mathrm{~b}$ shows $M$ vs. $T$ on LNMO7, LNMO8 and LNMO9 samples.

\section{Conclusions}

In conclusion, magnetic isotherms and the respective entropy change for samples belonging to the series of sodiumsubstituted lanthanum manganites LNMO6 to LNMO9 were evaluated. An appreciable change in magnetic entropy was observed for the samples and the entropy variation is dependent on magnetic field and temperature. From the entropy values of LNMO6 to LNMO9, it can be pointed out that entropy change increases with increase in magnetization while it exhibits maximum entropy change in the temperature range of 250 to $320 \mathrm{~K}$. Maximum entropy variation is observed for sample with lower $\mathrm{Na}$ content, which is LNMO6. Magnetic isotherms were also utilized to generate Arrott plots to study the variation of transition temperature. It was found that there is decrease in $T_{\mathrm{C}}$ with increase in Na concentration, which was confirmed from the $M v s . T$ measurements. Monovalent-substituted lanthanum manganites exhibit tunable magnetic properties and are potential candidates for magnetocaloric applications.

\section{Acknowledgements}

SN acknowledges DST INSPIRE (No. DST/INSPIRE Fellowship/2010/236) for the research fellowship. MRA acknowledges BRNS Project No. 2011/34/7/BRNS/0596 and IAA is grateful to Sultan Qaboos University for research Grant No. IG/SCI/PHYS/14/03. SN and MRA are also grateful to DST DAAD PPP programme.

\section{References}

1. Pollert E, Krupicka S and Kuzmicova E 1982 J. Phys. Chem. Solids $\mathbf{4 3} 1137$

2. Coey J M D and Viret M 1999 Adv. Phys. 48167

3. Roy S, Guo Y Q, Venkatesh S and Ali N 2001 J. Phys.: Condens. Matter 139547

4. Shimura Tetsuo, Hayashi Toshimasa, Inaguma Yoshiyuki and Itoh Mitsuru 1996 J. Solid State Chem. 124250

5. Gor'kov L P and Kresin V Z 2004 Phys. Rep. 400149
6. Gayathri N, Raychaudhuri A K and Tiwary S K 1997 Phys. Rev. B 561345

7. Tao Zou, Fen Wang, Yi Liu, Li-Qin Yan and Young Sun 2010 Appl. Phys. Lett. 97092501

8. Mozzati M C, Malavasi L, Azzoni C B and Flor G $2004 \mathrm{~J}$. Magn. Magn. Mater. 272-276 1579

9. Pecharsky V K and Gschneidner K A Jr 1999 J. Magn. Magn. Mater. 20044

10. Phana Manh-Huong and Yub Seong-Cho 2007 J. Magn. Magn. Mater. 308325

11. Franco V, Blazquez J S, Ingale B and Conde A 2012 Annu. Rev. Mater. Res. 42305

12. Koroleva L I and Morozov A S 2013 Am. J. Mod. Phys. 261

13. Szymczak R, Kolano R, Kolano-Burian A, Dyakonov V P and Szymczak H 2010 Acta Physica Polonica A 1117

14. Sande P, Hueso L E, Miguéns D R, Rivas J, Rivadulla F and Loépez-Quintela M A 2001 Appl. Phys. Lett. 7913

15. Sethulakshmi N, Al-Omari I A, Suresh K G and Anantharaman M R 2014 Appl. Phys. Lett. 104092407

16. Kalyana Lakshmi Y, Venkataiah $\mathrm{G}$ and Venugopal Reddy $\mathrm{P}$ 2009 J. App. Phys. 106023707

17. Thaljaoui R, Boujelben W, Pȩkała K, Pȩkała M, Cheikhrouhou-Koubaa W and Cheikhrouhou A 2013 J. Mater. Sci. 483894

18. Cheikh-Rouhou Koubaa W, Koubaa M and Cheikhrouhou A 2009 J. Mater. Sci. 441780

19. Cheikh-Rouhou Koubaa W, Koubaa M and Cheikhrouhou A 2009 J. Phys: Conference Series 153012046

20. Debnath J C, Zeng R, Kim J H and Dou S X 2011 J. Alloy. Comp. 5093699

21. Zhong W, Chen W, Ding W P, Zhang N, Hu A, Du Y W and Yan Q J 1998 Eur. Phys. J. B 3169

22. Sethulakshmi N, Unnimaya A N, Al-Omari I A, Al-Harthi S, Sagar S, Thomas S and Anantharaman M R 2015 J. Mag. Mag. Mater. 39175

23. Shivakumara C, Subbanna G N, Lalla N P and Hegde M S 2004 Mater. Res. Bull. 3971

24. Shivakumara C, Hegde M S, Srinivasa T, Vasanthacharya N Y, Subbanna G N and Lalla N P 2001 J. Mater. Chem. 112572

25. Ye S L, Song W H, Dai J M, Wang K Y, Wang S G, Dua J J, Sun Y P, Fang J, Chen J L and Gao B J 2001 J. Appl. Phys. 906

26. Kalyana Lakshmi Y, Venkataiah G, Vithal M and Venugopal Reddy P 2008 Physica B $\mathbf{4 0 3} 3059$

27. Rao G H, Sun J R, Bärner K and Hamad N 1999 J. Phys.: Condens. Matter 111523

28. Morrish A H 1965 The physical principles of magnetism (New York: Wiley), Chapter 3

29. Veena Gopalan E, Al-Omari I A, Sakthi Kumar D, Yoshida Y, Joy P A and Anantharaman M R 2010 Appl. Phys. A 99497

30. Zhong W, Cheng W, Ding W P, Zhang N, Du Y W and Yan Q J 1998 Solid State Commun. 10655

31. Bohigas X, Tejada J, Del Barco E, Zhang X X and Sales M 1998 Appl. Phys. Lett. 73390 